\title{
Review: Placental mitochondrial function and structure in gestational disorders
}

Olivia Holland $^{1 *}$, Marloes Dekker Nitert ${ }^{2,3}$, Linda A Gallo ${ }^{4,5}$, Meliha Vejzovic ${ }^{1}$, Joshua $\mathbf{J}$ Fisher $^{1}$, Anthony V Perkins ${ }^{1}$

${ }^{1}$ School of Medical Science, Menzies Health Institute Queensland, Griffith University, Gold Coast Campus Southport, Queensland, Australia.

${ }^{2}$ University of Queensland Centre for Clinical Research, Royal Brisbane and Women's Hospital, Queensland, Australia.

${ }^{3}$ School of Chemistry and Molecular Biosciences, The University of Queensland, Queensland, Australia.

${ }^{4}$ Mater Research Institute, The University of Queensland, Translational Research Institute, Queensland, Australia.

${ }^{5}$ School of Biomedical Sciences, The University of Queensland, Queensland, Australia.

*Corresponding author:

Olivia Holland

School of Medical Science, Menzies Health Institute Queensland, Griffith University Gold Coast Campus, Parklands Drive, Southport, Qld, 4222, Australia

Phone: + 61 (0) 7 55528970; Fax: + 61 (0) 7 56780795; e-mail: o.holland@griffith.edu.au 


\begin{abstract}
The aetiology of many gestational disorders is still unknown. However, insufficient transplacental nutrient and oxygen transfer due to abnormal placentation is characteristic of several pathologies, and may alter the function of placental mitochondria. Mitochondria are multifunctional organelles that respond to a wide range of stimuli - such as physiological changes in cellular energy demands or various pathologies - by reshaping via fusion or fission, increasing/decreasing in number, altering oxidative phosphorylation, and signalling cellular functions such as apoptosis. Mitochondrial function is integral to tissue functions including energy production, metabolism, and regulation of various cellular responses including response to oxidative stress. This review details the functions of placental mitochondria and investigates mitochondrial function and structure in gestational disorders including preeclampsia, intrauterine growth restriction, diabetes mellitus, and obesity. Placental mitochondrial dysfunction may be critical in a range of gestational disorders which have important implications for maternal and fetal/offspring health.
\end{abstract}

Keywords: mitochondria; oxidative stress; preeclampsia; intrauterine growth restriction; diabetes; obesity 


\section{Introduction}

Mitochondria originated from the symbiosis of primordial eukaryotic cells and aerobic bacteria. Mitochondria, which contain their own genome (mtDNA) and machinery to synthesise RNA and proteins, work in concert with the nuclear genome and other organelles. Almost all cellular energy is produced through oxidative phosphorylation in mitochondria; partnered redox reactions transfer electrons through oxygen to water and pump protons into the mitochondrial inner membrane through respiratory complexes (complexes I, III, and IV). The electrochemical gradient created by the transfer of protons is termed mitochondrial membrane potential $(\Delta \Psi \mathrm{m})$, and is harnessed by ATP synthase (complex V) to generate ATP. Mitochondria are known as the powerhouses of the cell due to their central role in ATP generation. However, mitochondria have several additional functions; they provide important signalling on cellular homeostasis, and are key regulators of cell fate through autophagy/apoptosis. Mitochondria form a dynamic reticulum, and the reshaping of this reticulum in response to differences in mitochondrial membrane potential helps control mitochondrial and cellular fate (Figure 1). In conjunction with the endoplasmic reticulum, mitochondria can regulate mediators of cell death such as calcium levels and caspases. Additionally, in the placenta and other tissues such as the adrenal glands, mitochondria are crucial to the production of steroids $[1,2]$.

Mitochondrial dysfunction is thought to contribute to a wide range of disorders related to oxidative stress, such as cardiovascular disease, type 2 diabetes, and neurodegenerative disorders. Partial occlusion of blood flow leading to local hypoxia is a common feature in several pathologies which show effects on the mitochondria. Mitochondria consume oxygen to generate ATP via oxidative phosphorylation, producing reactive oxygen species (ROS) as 
a by-product. Oxygen variability can lead to oxidative stress when there is a disproportionately high production of ROS in comparison to antioxidants [3, 4].

Mitochondria are susceptible to damage by these free radicals, which may result in alterations in their structure and function [5].

Pregnancy itself is characterised by increased oxidative stress, which is often heightened in disorders. Of relevance to this review, increased placental oxidative stress is a feature of several gestational pathologies including preeclampsia, intrauterine growth restriction (IUGR), maternal diabetes, and maternal obesity [6]. Preeclampsia and IUGR are associated with reduced placental perfusion, potentially leading to oxygen deprivation [7]. Placentae afflicted by maternal diabetes and/or obesity are exposed to a range of insults, including high glucose and fatty acid levels as well as inflammatory mediators. These insults may lead to abnormal function of the uteroplacental unit, including impaired placentation [8]. As a number of pregnancy pathologies share a mutual phenotype of restricted or heightened variability in placental oxygen supply [9], which is likely to alter mitochondrial structure and function $[10,11]$, placental mitochondria may be aetiologically important in several pregnancy pathologies.

This review details important features of placental mitochondria and summarises evidence on how placental mitochondria are affected in a range of pregnancy disorders. Gaining a greater appreciation of mitochondrial content, structure, and function in the placenta provides an opportunity to explore interventional avenues. 


\section{Mitochondria reactive oxygen species}

Oxidative stress mediated by ROS is a common feature of several gestational disorders. Mitochondria are the main sites of ROS generation, and are also susceptible to ROS-mediated damage. The generation of ROS result from the transfer of a single electron from a redox donor to molecular oxygen, yielding superoxide which can be converted to hydrogen peroxide by superoxide dismutase. This often occurs when oxygen reacts with electrons generated by complex I and III but can also occur at complex II of the electron transport chain [12]. Approximately $0.15-4 \%$ of oxygen in mitochondria produces ROS [13]. Hydrogen peroxide can alter protein structure and function through altering the redox state of thiol moieties in sensitive proteins [14].The amount of ROS produced is dependent on mitochondrial characteristics such as activity level and dynamics as well as the type and amount of available fuel (carbohydrate, lipid, or amino acid) [15].

The production of ROS is physiological and ROS regulate many cellular functions including autophagy, anti-microbial effects, and act as signalling molecules in many pathways including cellular differentiation and inflammation [16]. ROS production also regulates mitochondrial fission and fusion in healthy cells, providing a mechanism that regulates mitochondrial morphology and function that is dependent on the redox state [17]. Excessive ROS production can, however, be detrimental to cellular function, causing oxidative damage to DNA, proteins and (membrane) lipids, which is associated with hypertension and insulin resistance [5]. Chronic oxidative stress can also lead to changes characteristic of senescence, and senescence of the syncytiotrophoblast may be a feature of the normal progression of pregnancy which is exaggerated in pathologies [18]. In the endothelium, excessive ROS production can affect vasodilation through the inhibition of the expression and function of 
endothelial nitric oxide synthase [19]. This regulatory effect of ROS on vasodilation may be involved in the pathogenesis of preeclampsia [20].

\section{Mitochondrial regulation of apoptosis}

Mitochondria are key signalling organelles due to their responsiveness to the metabolic functioning of the cell. The interactions between mitochondria and the endoplasmic reticulum are critical to cell homeostasis and signalling (reviewed by [18]). Mitochondria can initiate apoptosis by the release of mitochondrial intermembrane space proteins such as cytochrome c into the cytoplasm through mitochondrial membrane permeabilization or rupture [21]. An early event in the initiation of apoptosis is the opening of the mitochondrial permeability transition pore and subsequent swelling of the mitochondrial matrix leading to rupture [21].

Swelling of mitochondria characteristic of apoptosis has been reported in isolated preeclamptic placental mitochondria [22] and similar morphological alterations of placental mitochondria are found in instances of gestational diabetes mellitus (GDM) [23]. Further, mitochondrial size appears to be reduced in preeclampsia [24], and the levels of many apoptotic proteins are altered in preeclamptic placentae [25]. In vivo or in vitro treatments with preeclampsia-associated factors have been shown to alter placental mitochondria and potentially lead to some of the perturbations seen in preeclampsia. An increase in soluble fms-like tyrosine kinase 1 (sFlt-1) is found in the maternal circulation in preeclampsia, and is thought to be involved in the inhibition of angiogenesis by reducing the circulating levels of proangiogenic factors such as vascular endothelial growth factor [26]. The administration of sFlt-1 to pregnant mice led to features characteristic of preeclampsia (hypertension and proteinuria), as well as increased oxidative stress, swollen mitochondria, and increased 
apoptosis in the placentae [27]. The authors suggest that sFlt-1 is involved in increased oxidative stress and the activation of the mitochondrial apoptotic pathway [27]. Antiphospholipid antibodies (aPLs) are an important maternal predisposing factor for the development of preeclampsia, although their mechanism of action is not well understood [2830]. c [31]. In vitro studies have demonstrated that aPLs are internalised by the syncytiotrophoblast and lead to multiple effects on syncytiotrophoblast mitochondria, including increased release of cytochrome c, depressed respiration, and changes in the expression of mitochondrial/apoptotic proteins [29]. Therefore, aPLs may primarily affect placental mitochondria, leading to the aberrant placental cell death and subsequent maternal immune activation that is characteristic of preeclampsia [32]. Further, rats exposed to a foodrestricted diet exhibited increased expression of pro-apoptotic proteins and cytochrome c release, indicating that maternal undernutrition also enhances mitochondria-dependent apoptosis in the placenta [33]. Therefore, multiple perturbations can lead to dysfunction of placental mitochondria and the induction of apoptosis.

It should be noted that although the role of mitochondria in apoptosis is well characterised in many tissues, the progression of apoptosis in the syncytiotrophoblast is not fully understood. The syncytiotrophoblast lacks cell borders, and it has been suggested that apoptosis cannot progress in a syncytium as in mononuclear cells because of the danger of continued uncontrolled cell death [18].The pro-apoptotic proteins, p53, BCL2 associated X apoptosis regulator, and cytochrome $\mathrm{c}$, have been reported to be decreased in syncytiotrophoblast mitochondria relative to the cytotrophoblast mitochondria from which they are derived [34]. Further, apoptosis is an active process requiring energy, and syncytiotrophoblast mitochondria appear to have reduced metabolic efficacy and ATP production [34]. Therefore, 
mitochondria in the syncytiotrophoblast may not regulate apoptosis in the same manner as other cell lineages.

\section{Mitochondrial content}

Cells contain multiple mitochondria arranged in a dynamic interconnected reticulum. The mitochondrial content or mass in cells is plastic and able to respond to a wide variety of stimuli such as caloric restriction, increased energy demands, and various disease states [3538]. In the placenta, pregnancy pathologies related to placental insufficiency including IUGR and preeclampsia, as well as maternal diabetes and obesity, are associated with changes in mitochondrial content (Table 1). Further, levels of mtDNA in the maternal circulation can be increased in preeclampsia and placental abruption, and this material is likely to be derived from the placenta $[39,40]$. A common feature of these conditions is increased oxidative stress. Hypoxic conditions are thought to occur in IUGR due to placental insufficiency and the subsequent reduction in placental blood flow [41]. Hypoxic stress can stimulate mitochondrial biogenesis and lead to increased mitochondrial content $[11,42,43]$.This protective mechanism may help meet metabolic demands by increasing the bioenergetic capacity of the tissue. Lower $\mathrm{pO}_{2}$ levels have been found in both the umbilical vein and artery in IUGR, indicative of a hypoxic fetal environment [44].

Mitochondrial content was found to be decreased or increased in the same pregnancy pathology by different studies (Table 1). In cardiac tissue, decreased mitochondrial content has been linked to ischemic insult and related tissue damage [45]. Conversely, increased mitochondrial content has been associated with hypoxia and oxidative stress in cardiac, pulmonary, hepatic, and neuronal cells [42, 46, 47]. In pathologies, proliferation of 
mitochondria is thought to occur as a compensatory mechanism for the disruption of cellular bioenergetics [42, 48]. However, increased placental mitochondrial ROS may directly damage mtDNA, thus inhibiting the adaptive biogenesis of the self-replicating mitochondria and reducing respiratory activity [49]. The apparent differences in response within the same pathologies may be linked to the severity or timing of the insult and the subsequent ability of the tissue to adapt through increased mitochondrial content. Either increased or decreased mitochondrial biogenesis could occur in an attempt to maintain normal fetal growth, with the effect depending on whether there is a compensatory response to increase energy output in nutritionally perturbed environments [44, 50, 51]. Vishnyakova et al. (2016) found increased placental mitochondrial content (mtDNA relative to nDNA) in early-onset but not late-onset preeclampsia, suggesting that the different pathophysiology leads to differences in mitochondrial response [52].

In the majority of placental investigations, tissue as a whole has been considered. However, mitochondria within different cell lineages often have distinct functions and are likely to respond differently to stimuli. In particular, mitochondria within two of the major placental cell types, cytotrophoblasts and the syncytiotrophoblast, have very different structure and roles $[34,53]$. Further, the antioxidant capacity of different regions of the placenta is varied, meaning that their ability to respond to hypoxia/reperfusion will be different [54]. In IUGR, Mando et al. (2014) found increased mitochondrial content in whole tissue but decreased content in cytotrophoblasts, indicating that the increased placental mitochondrial content is due to other cell lineages. The syncytiotrophoblast is in direct contact with maternal blood and has been suggested to be the cell type most affected in IUGR and preeclampsia [55]. The syncytiotrophoblast possess low levels of antioxidant enzymes, and mitochondria with reduced membrane potential and increased hydrogen peroxide production compared to the 
cytotrophoblasts which fuse to form the syncytiotrophoblast $[34,54]$. Therefore, syncytiotrophoblast mitochondria may be more affected by hypoxia/reperfusion and could be the source of the increased mitochondrial content; however, the mechanisms regulating mitochondrial dynamics in the syncytiotrophoblast are not well characterised.

Mitochondrial biogenesis is controlled by multiple transcription factors which include nuclear respiratory factor 1 (NRF1), mitochondrial transcription factor A (TFAM), B1 (TFB1M), and B2 (TFB2M) (Figure 2). In addition, the co-activator peroxisome proliferator activated receptor $\gamma$ coactivator- 1 alpha (PGC-1 $\alpha)$ is an important stimulator of mitochondrial transcription. PGC-1 $\alpha$ stimulates mitochondrial biogenesis through the induction of NRF1, which in turn increases TFAM. Mitochondrial transcription factors TFB1M and TFB2M also interact with TFAM and mitochondrial RNA polymerase to support transcription [56]. Several studies have linked mitochondrial biogenesis transcription factors to placental mitochondrial content or pathology. In placentae with IUGR and/or preeclampsia where mitochondrial content was reduced, mRNA expression of PGC-1 $\alpha$ and NRF1 was also decreased [51, 57]. Where IUGR was shown to increase mitochondrial content, NRF1 expression was also increased [51]. Further, maternal caloric restriction in a rodent IUGR model leads to increased placental mitochondria content and the upregulation of biogenesis markers [58], whereas PGC-1 $\alpha$ appears to be decreased in the placentae of rats subjected to reduced uterine perfusion pressure [59].

The regulation of mitochondrial dynamics occurs though mitochondrial biogenesis, and continuous cycles of fission and fusion. These processes are thought to target damaged/depolarized mitochondria for autophagy [60]. Vishnyakova et al. (2016) reported increased mitochondrial content in preeclamptic placentae without increased NRF1, as well 
as lower TFAM protein expression. However, there was a significant increase in the mitochondrial fusion regulator, optic atrophy 1 mitochondrial dynamin like GTPase (OPA1), suggesting mitochondrial fusion as a mechanism for increased content, potentially by stabilisation of mitochondrial structures [52]. Indeed, overexpression of OPA1 protects from ischemia in the heart and brain, and ROS production, cytochrome c release, and apoptosis [61].

\section{Syncytiotrophoblast mitochondria}

As well as generating cellular energy in the form of ATP from oxidative phosphorylation, mitochondria are important in the synthesis of steroid hormones (reviewed in the placenta by [2]). In the human placenta, the syncytiotrophoblast forms the interface between maternal and fetal systems. Progesterone synthesised by the syncytiotrophoblast from maternally-derived cholesterol is central to the establishment and maintenance of the pregnancy. Progesterone functions in modulation of the endometrium and maternal immune response to fetal factors, and decreased progesterone levels are associated with spontaneous abortion/miscarriage [62]. The multinucleate syncytiotrophoblast is formed by the fusion of underlying mononuclear cytotrophoblasts. During differentiation into the syncytiotrophoblast, mitochondria appear to become highly specialised for steroidogenesis.

The production of progesterone requires the transport of cholesterol to the mitochondria and cleavage of the cholesterol side-chain. Unlike the mitochondria of cytotrophoblasts, syncytiotrophoblast mitochondria contain high levels of cytochrome P450scc [53], which is responsible for the conversion of cholesterol into pregnenolone in the inner mitochondrial membrane. Therefore, mitochondria acquire steroidogenic ability during the formation of the 
syncytiotrophoblast. Steroidogenesis requires mitochondrial contact sites involving multiprotein systems, where outer and inner membranes are in close proximity. In other steroidogenic tissues (e.g. adrenal glands and gonads), intracellular transport of cholesterol to the mitochondria is regulated via the mitochondrial sterol carrier protein steroidogenic acute regulatory protein $(\mathrm{StAR})[1,2]$. StAR is not expressed in the placenta and it has been suggested that the regulation of steroidogenesis is through the structurally-related protein StAR related lipid transfer domain containing 3 (STARD3; MLN64) [1, 2, 53]. In addition, the mitochondrial heat shock protein (HSP) HSP60 has been shown to associate with STARD3 in the placenta and may participate in steroidogenesis [63]. HSP have known functions in the cellular response to stress, often as chaperones; however, HSP can have additional roles. Antibodies against HSP60 are associated with various autoimmune conditions and increased serum cholesterol in atherosclerosis, indicating that HSP60 may have a role in cholesterol transport [63-65]. Therefore, cholesterol transport in placental mitochondria appears to utilise tissue-specific systems.

Syncytiotrophoblast mitochondria are smaller and more irregular in shape than those of the cytotrophoblasts, and also have atypical cristae morphology (Figure 3), potentially through reduced dimerization of ATP synthase which helps to form mitochondrial architecture [53, 66]. It has been suggested that these morphological changes are related to steroidogenesis, as cholesterol could be more efficiently transported to P450scc in the inner mitochondrial membrane in smaller mitochondria [66].

Syncytiotrophoblast mitochondria have been reported to have a reduced coupling control of oxidative phosphorylation to ATP production in comparison to cytotrophoblast mitochondria, as well as reduced cardiolipin content, which is important in efficient oxidative 
phosphorylation [34]. Syncytiotrophoblast mitochondria also have reduced membrane potential and increased levels of hydrogen peroxide [34]. P450scc, which is present in high levels in the syncytiotrophoblast, is involved in superoxide generation and may be source of oxygen radicals in syncytiotrophoblast mitochondria [34, 67].

The fusion of cytotrophoblasts into a syncytium is linked to early stages of the apoptotic cascade, with apoptosis-related proteins such as caspase 8 required for fusion [68].

Regulation of the progression of the apoptotic cascade appears to occur at the mitochondrial level and involve members of the anti-apoptotic BCL2 family [69]. There is also evidence that mitochondria are directly involved in the differentiation of cytotrophoblasts into the syncytiotrophoblast. In primary villous cytotrophoblasts, inhibition of the mitochondrial respiratory chain leads to a decrease in cell fusion and hormone production (human chorionic gonadotropin and leptin). Lactate production also appears to be transiently increased during cytotrophoblast differentiation, suggesting that anaerobic metabolism is important during differentiation [70].

\section{Mitochondria in preeclampsia and intrauterine growth restriction}

Preeclampsia is a hypertensive disorder of pregnancy characterised by maternal endothelial dysfunction. Both preeclampsia and IUGR are associated with reduced/intermittent placental perfusion and increased oxidative stress $[3,71]$. As detailed earlier in this review, preeclampsia and IUGR can lead to changes in placental apoptosis, mitochondrial fission/fusion, and mitochondrial content (Table 1). Additionally, proteomic analysis of placental tissue from preeclamptic pregnancies shows the involvement of multiple mitochondrial-related functions including the tricarboxylic acid cycle, electron transport 
chain, fatty acid oxidation, ATP binding, $\mathrm{Ca}^{2+}$ binding, apoptosis, HSP70, and the mitochondrial antioxidant protein peroxiredoxin III/SP-22 [22, 71-73]. The mitochondria of preeclamptic placentae also exhibit swelling and damaged cristae, as well as reduced expression/activity of mitochondrial complexes and ATP synthase [74-76]. A reduction in electron flow through complex III and possible damage to other complexes may contribute to the excess ROS production seen in preeclampsia [74]. Mitochondrial respiration is increased in early-onset preeclampsia and these mitochondria are also less sensitive to $\mathrm{Ca}^{2+}$ depolarization, suggesting an adaptive response to oxidative stress [52]. $\mathrm{Ca}^{2+}$ levels are important in cellular homeostasis and $\mathrm{Ca}^{2+}$ is a critical signalling molecule between mitochondria and the endoplasmic reticulum [18]. Preeclampsia has been with associated alterations in intracellular $\mathrm{Ca}^{2+}$ which may affect apoptosis in the placenta [76]. IUGR independent of preeclampsia leads to changes in mitochondrial content (Table 1), and also increased mitochondrial respiration in cytotrophoblasts [51]. Maternal food restriction resulting in fetal growth retardation can lead to changes in placental mitochondrial proteins, and mitochondria from these placentae have increased oxygen consumption but fail to maintain ATP production [58].

\section{Mitochondria in maternal diabetes}

High circulating glucose concentrations in maternal diabetes, which includes both preexisting diabetes and GDM, may adversely impact on placental function. In individuals with diabetes, there are ample studies reporting alterations in mitochondrial content, respiratory function, and complex activity in non-gestational tissues. However, studies examining specific mitochondrial changes in the placenta are limited. In whole placentae from women with type 1 diabetes, activity of complexes I and III were reduced and, in type 2 diabetes, 
combined activity of complexes II and III were reduced [49]. In the same study, the level of mitochondrial hydrogen peroxide in placentae was elevated in type 1 diabetes only. This aligns with the reductions seen in complex I activity, in being a major source of ROS production. Placental mitochondrial content remained unaffected in both types of diabetes, which differs from previous studies in other gestational pathologies, including reduced mtDNA in preeclampsia and IUGR [51] (Table 1). Previously, Qiu et al. reported that placental mtDNA copy number was positively associated with a marker of oxidative stress, 8hydroxy-2' -deoxyguanosine, in both uncomplicated and GDM pregnancies [77]. There was no difference in this relationship between non-GDM and GDM placentae, but this study was carried out in only 40 women and may have been underpowered. In villous tissue from GDM pregnancies, the expression of mitochondrial complexes was also significantly reduced, but varied depending on whether women had been treated with diet alone or either insulin or glyburide [78]. Regardless of treatment, protein expression of PGC1 $1 \alpha$, the master regulator of mitochondrial biogenesis, and PPAR $\gamma$, an important regulator of fatty acid oxidation, were reduced in placentae from these women with GDM. In a study that examined placental ultrastructure from 10 women with GDM, mitochondria were found to be swollen or completely destroyed, and architecturally disrupted [23]. In rats rendered mildly diabetic, using streptozotocin prior to mating, an increase in mitochondrial membrane fluidity was observed in placental tissue, along with increases in the ratio of unsaturated to saturated fatty acids [79]. This was associated with an increase in both placental and fetal weights; however, a direct cause and effect relationship remains to be tested. Indeed, in humans, placental lipid metabolism is known to be altered in maternal diabetes [80], but larger cohort studies are required to clarify the association between placental mitochondrial content, structure, and function, and adverse fetal outcomes. 


\section{Mitochondria in obesity}

Obesity is associated with excess circulating fatty acids, which can affect placental mitochondrial function [81]. Mitochondrial content, as measured in mitochondrial DNA amount, is decreased in placentas from obese women, although when measured by citrate synthase activity, is unaltered [49]. This suggests that despite a lower mitochondrial number, the oxidative capacity of the mitochondria to produce ATP remains the same.

The lower mitochondrial content is, however, associated with lower complex I (but not complex II, III, or IV) activity but higher mitochondrial ROS production [49]. Placental villous tissues from overweight and obese women have a 6 and 14-fold increase in ROS production [82] and to a similar extent in male and female offspring. Placental cellular ATP production, a marker of mitochondrial function, decreases with maternal obesity [82]. This may be due to decreased placental mitochondrial content as well as reduced expression of complex I-IV, and this is associated with a decrease in mitochondrial function as measured by respiration [82]. Similarly, in the placental cell line Swan 71, incubation with palmitate stimulates overall cellular ROS production as well as mitochondrial ROS production resulting in reduced secretion of IL-1 $\beta$, IL-6, and IL-8 [81]. The increases in mitochondrial ROS production in obesity may result in damaged mitochondrial DNA and thereby decreased mitochondrial content. Placental mitochondrial content and function have high interindividual variability in both lean and obese women [49] whilst unclear, (epi)genetic makeup, infant gender, and levels of insulin resistance could contribute to the variability. 


\section{Conclusion}

Mitochondria are critical to cellular viability, and mitochondrial function can be disturbed by variability in oxygen supply. Reduced/interment blood flow to the placenta resulting in oxidative stress is thought to be a common feature of several pregnancy complications, and this oxidative stress is likely to affect placental mitochondria. Indeed, placental mitochondrial function is altered in a number of pregnancy disorders. Whilst the majority of studies observed an increase in oxidative stress, the subsequent mitochondrial damage, mitochondrial bioenergetics, and adaptive responses varied even within the same pathology. Although the pathophysiology underlying various gestational disorders may be different, alterations in mitochondrial function and structure are a common terminal pathway and may offer avenues for the development of therapeutics. However, the mechanisms controlling the mitochondrial response to stress are complex and require further investigation.

\section{Conflicts of interest}

The authors have no conflicts of interest to declare.

\section{Acknowledgements}

This review was generated as part of the Queensland Perinatal Consortium Inaugural Conference held on July 15th 2016 in Brisbane, Queensland Australia. The conference was supported by an Intra-Faculty Collaborative Workshop grant from the Faculty of Medicine and Biomedical Sciences, The University of Queensland. 


\section{References}

[1] Miller WL. Steroid hormone synthesis in mitochondria. Mol Cell Endocrinol. 2013;379(1-2):62-73.

[2] Martinez F, Olvera-Sanchez S, Esparza-Perusquia M, Gomez-Chang E and Flores-Herrera O.

Multiple functions of syncytiotrophoblast mitochondria. Steroids. 2015;103:11-22.

[3] Myatt L and Cui X. Oxidative stress in the placenta. Histochem Cell Biol. 2004;122(4):369-82.

[4] Turrens JF. Mitochondrial formation of reactive oxygen species. J Physiol. 2003;552(Pt 2):335-44.

[5] Bindoli A. Lipid peroxidation in mitochondria. Free Radic Biol Med. 1988;5(4):247-61.

[6] Gupta S, Agarwal A and Sharma RK. The role of placental oxidative stress and lipid peroxidation in preeclampsia. Obstet Gynecol Surv. 2005;60(12):807-16.

[7] Burton GJ. Oxygen, the Janus gas; its effects on human placental development and function. Journal of anatomy. 2009;215(1):27-35.

[8] Desoye $\mathrm{G}$ and Hauguel-de Mouzon S. The human placenta in gestational diabetes mellitus. The insulin and cytokine network. Diabetes Care. 2007;30 Suppl 2:S120-6.

[9] Gagnon R. Placental insufficiency and its consequences. Eur J Obstet Gynecol Reprod Biol. 2003;110 Suppl 1:S99-107.

[10] Colleoni F, Padmanabhan N, Yung HW, Watson ED, Cetin I, Tissot van Patot MC, Burton GJ and Murray AJ. Suppression of mitochondrial electron transport chain function in the hypoxic human placenta: a role for miRNA-210 and protein synthesis inhibition. PLoS One. 2013;8(1):e55194.

[11] Hung TH, Skepper JN, Charnock-Jones DS and Burton GJ. Hypoxia-reoxygenation: a potent inducer of apoptotic changes in the human placenta and possible etiological factor in preeclampsia. Circ Res. 2002;90(12):1274-81.

[12] Quinlan CL, Perevoshchikova IV, Hey-Mogensen M, Orr AL and Brand MD. Sites of reactive oxygen species generation by mitochondria oxidizing different substrates. Redox biology.

2013;1(1):304-12.

[13] Brand MD. The sites and topology of mitochondrial superoxide production. Exp Gerontol. 2010;45(7-8):466-72.

[14] Go YM, Chandler JD and Jones DP. The cysteine proteome. Free Radic Biol Med. 2015;84:22745.

[15] Gorlach A, Dimova EY, Petry A, Martinez-Ruiz A, Hernansanz-Agustin P, Rolo AP, Palmeira CM and Kietzmann T. Reactive oxygen species, nutrition, hypoxia and diseases: Problems solved? Redox Biol. 2015;6:372-85.

[16] Dan Dunn J, Alvarez LA, Zhang $X$ and Soldati T. Reactive oxygen species and mitochondria: A nexus of cellular homeostasis. Redox Biol. 2015;6:472-85.

[17] Willems PH, Rossignol R, Dieteren CE, Murphy MP and Koopman WJ. Redox Homeostasis and Mitochondrial Dynamics. Cell Metab. 2015;22(2):207-18.

[18] Burton GJ, Yung HW and Murray AJ. Mitochondrial - Endoplasmic reticulum interactions in the trophoblast: Stress and senescence. Placenta. 2016.

[19] Farrow KN, Lakshminrusimha S, Reda WJ, Wedgwood S, Czech L, Gugino SF, Davis JM, Russell JA and Steinhorn RH. Superoxide dismutase restores eNOS expression and function in resistance pulmonary arteries from neonatal lambs with persistent pulmonary hypertension. Am J Physiol Lung Cell Mol Physiol. 2008;295(6):L979-87.

[20] Matsubara K, Higaki T, Matsubara Y and Nawa A. Nitric oxide and reactive oxygen species in the pathogenesis of preeclampsia. Int J Mol Sci. 2015;16(3):4600-14.

[21] Sesso A, Belizario JE, Marques MM, Higuchi ML, Schumacher RI, Colquhoun A, Ito E and Kawakami J. Mitochondrial swelling and incipient outer membrane rupture in preapoptotic and apoptotic cells. Anat Rec (Hoboken). 2012;295(10):1647-59.

[22] Shi Z, Long W, Zhao C, Guo X, Shen R and Ding H. Comparative proteomics analysis suggests that placental mitochondria are involved in the development of pre-eclampsia. PLoS One.

2013;8(5):e64351. 
[23] Meng Q, Shao L, Luo X, Mu Y, Xu W, Gao C, Gao L, Liu J and Cui Y. Ultrastructure of Placenta of Gravidas with Gestational Diabetes Mellitus. Obstet Gynecol Int. 2015;2015:283124.

[24] Zsengellér ZK, Rajakumar A, Hunter JT, Salahuddin S, Rana S, Stillman IE and Karumanchi SA. Trophoblast mitochondrial function is impaired in preeclampsia and correlates negatively with the expression of soluble fms-like tyrosine kinase 1. Pregnancy Hypertension: An International Journal of Women's Cardiovascular Health. 2016.

[25] Cali U, Cavkaytar S, Sirvan L and Danisman N. Placental apoptosis in preeclampsia, intrauterine growth retardation, and HELLP syndrome: an immunohistochemical study with caspase-3 and bcl-2. Clin Exp Obstet Gynecol. 2013;40(1):45-8.

[26] Hiratsuka S, Maru Y, Okada A, Seiki M, Noda T and Shibuya M. Involvement of Flt-1 tyrosine kinase (vascular endothelial growth factor receptor-1) in pathological angiogenesis. Cancer Res. 2001;61(3):1207-13.

[27] Jiang Z, Zou Y, Ge Z, Zuo Q, Huang SY and Sun L. A Role of sFlt-1 in Oxidative Stress and Apoptosis in Human and Mouse Pre-Eclamptic Trophoblasts. Biol Reprod. 2015;93(3):73.

[28] Duckitt K and Harrington D. Risk factors for pre-eclampsia at antenatal booking: systematic review of controlled studies. Bmj. 2005;330(7491):565.

[29] Pantham P, Viall CA, Chen Q, Kleffmann T, Print CG and Chamley LW. Antiphospholipid antibodies bind syncytiotrophoblast mitochondria and alter the proteome of extruded syncytial nuclear aggregates. Placenta. 2015;36(12):1463-73.

[30] Tong M, Viall CA and Chamley LW. Antiphospholipid antibodies and the placenta: a systematic review of their in vitro effects and modulation by treatment. Human reproduction update.

2015;21(1):97-118.

[31] Viall CA and Chamley LW. Histopathology in the placentae of women with antiphospholipid antibodies: A systematic review of the literature. Autoimmun Rev. 2015;14(5):446-71.

[32] Viall CA, Chen Q, Stone PR and Chamley LW. Human extravillous trophoblasts bind but do not internalize antiphospholipid antibodies. Placenta. 2016;42:9-16.

[33] Belkacemi L, Desai M, Nelson DM and Ross MG. Altered mitochondrial apoptotic pathway in placentas from undernourished rat gestations. Am J Physiol Regul Integr Comp Physiol.

2011;301(6):R1599-615.

[34] Bustamante J, Ramirez-Velez R, Czerniczyniec A, Cicerchia D, Aguilar de Plata AC and LoresArnaiz S. Oxygen metabolism in human placenta mitochondria. J Bioenerg Biomembr.

2014;46(6):459-69.

[35] Civitarese AE, Carling S, Heilbronn LK, Hulver MH, Ukropcova B, Deutsch WA, Smith SR and Ravussin E. Calorie restriction increases muscle mitochondrial biogenesis in healthy humans. PLoS Med. 2007;4(3):e76.

[36] Hood DA, Tryon LD, Carter HN, Kim Y and Chen CC. Unravelling the mechanisms regulating muscle mitochondrial biogenesis. Biochem J. 2016;473(15):2295-314.

[37] Holloszy JO. Biochemical adaptations in muscle. Effects of exercise on mitochondrial oxygen uptake and respiratory enzyme activity in skeletal muscle. J Biol Chem. 1967;242(9):2278-82.

[38] Liesa M, Palacin M and Zorzano A. Mitochondrial dynamics in mammalian health and disease. Physiol Rev. 2009;89(3):799-845.

[39] Qiu C, Hevner K, Enquobahrie DA and Williams MA. A case-control study of maternal blood mitochondrial DNA copy number and preeclampsia risk. International journal of molecular epidemiology and genetics. 2012;3(3):237-44.

[40] Williams MA, Sanchez SE, Ananth CV, Hevner K, Qiu C and Enquobahrie DA. Maternal blood mitochondrial DNA copy number and placental abruption risk: results from a preliminary study. International journal of molecular epidemiology and genetics. 2013;4(2):120-7.

[41] Jansson T and Powell TL. IFPA 2005 Award in Placentology Lecture. Human placental transport in altered fetal growth: does the placenta function as a nutrient sensor? -- a review. Placenta.

2006;27 Suppl A:S91-7. 
[42] Lee HC, Yin PH, Lu CY, Chi CW and Wei YH. Increase of mitochondria and mitochondrial DNA in response to oxidative stress in human cells. Biochem J. 2000;348 Pt 2:425-32.

[43] Gutsaeva DR, Carraway MS, Suliman HB, Demchenko IT, Shitara H, Yonekawa H and Piantadosi CA. Transient hypoxia stimulates mitochondrial biogenesis in brain subcortex by a neuronal nitric oxide synthase-dependent mechanism. J Neurosci. 2008;28(9):2015-24.

[44] Lattuada D, Colleoni F, Martinelli A, Garretto A, Magni R, Radaelli T and Cetin I. Higher mitochondrial DNA content in human IUGR placenta. Placenta. 2008;29(12):1029-33.

[45] Bertholet AM, Delerue T, Millet AM, Moulis MF, David C, Daloyau M, Arnaune-Pelloquin L, Davezac N, Mils V, Miquel MC, Rojo M and Belenguer P. Mitochondrial fusion/fission dynamics in neurodegeneration and neuronal plasticity. Neurobiol Dis. 2016;90:3-19.

[46] Lee HM, Greeley GH, Jr. and Englander EW. Sustained hypoxia modulates mitochondrial DNA content in the neonatal rat brain. Free Radic Biol Med. 2008;44(5):807-14.

[47] Costa LE, Boveris A, Koch OR and Taquini AC. Liver and heart mitochondria in rats submitted to chronic hypobaric hypoxia. Am J Physiol. 1988;255(1 Pt 1):C123-9.

[48] Sitarz KS, Yu-Wai-Man P, Pyle A, Stewart JD, Rautenstrauss B, Seeman P, Reilly MM, Horvath R and Chinnery PF. MFN2 mutations cause compensatory mitochondrial DNA proliferation. Brain.

2012;135(Pt 8):e219, 1-3; author reply e20, 1-3.

[49] Hastie R and Lappas M. The effect of pre-existing maternal obesity and diabetes on placental mitochondrial content and electron transport chain activity. Placenta. 2014;35(9):673-83.

[50] Cetin I and Alvino G. Intrauterine growth restriction: implications for placental metabolism and transport. A review. Placenta. 2009;30 Suppl A:S77-82.

[51] Mando C, De Palma C, Stampalija T, Anelli GM, Figus M, Novielli C, Parisi F, Clementi E, Ferrazzi E and Cetin I. Placental mitochondrial content and function in intrauterine growth restriction and preeclampsia. Am J Physiol Endocrinol Metab. 2014;306(4):E404-13.

[52] Vishnyakova PA, Volodina MA, Tarasova NV, Marey MV, Tsvirkun DV, Vavina OV, Khodzhaeva ZS, Kan NE, Menon R, Vysokikh MY and Sukhikh GT. Mitochondrial role in adaptive response to stress conditions in preeclampsia. Sci Rep. 2016;6:32410.

[53] Martinez F, Kiriakidou M and Strauss JF, 3rd. Structural and functional changes in mitochondria associated with trophoblast differentiation: methods to isolate enriched preparations of syncytiotrophoblast mitochondria. Endocrinology. 1997;138(5):2172-83.

[54] Watson AL, Skepper JN, Jauniaux E and Burton GJ. Susceptibility of human placental syncytiotrophoblastic mitochondria to oxygen-mediated damage in relation to gestational age. J Clin Endocrinol Metab. 1998;83(5):1697-705.

[55] Scifres CM and Nelson DM. Intrauterine growth restriction, human placental development and trophoblast cell death. J Physiol. 2009;587(Pt 14):3453-8.

[56] Ventura-Clapier R, Garnier A and Veksler V. Transcriptional control of mitochondrial biogenesis: the central role of PGC-1alpha. Cardiovascular research. 2008;79(2):208-17.

[57] Poidatz D, Dos Santos E, Duval F, Moindjie H, Serazin V, Vialard F, De Mazancourt P and Dieudonne $\mathrm{MN}$. Involvement of estrogen-related receptor-gamma and mitochondrial content in intrauterine growth restriction and preeclampsia. Fertil Steril. 2015;104(2):483-90.

[58] Mayeur S, Lancel S, Theys N, Lukaszewski MA, Duban-Deweer S, Bastide B, Hachani J, Cecchelli R, Breton C, Gabory A, Storme L, Reusens B, Junien C, Vieau D and Lesage J. Maternal calorie restriction modulates placental mitochondrial biogenesis and bioenergetic efficiency: putative involvement in fetoplacental growth defects in rats. Am J Physiol Endocrinol Metab.

2013;304(1):E14-22.

[59] Delany A, McCarthy F, Walsh S and Kenny L. PP053. The role of peroxisome proliferatoractivated receptor gamma co-activator 1-alpha in pregnancy. Pregnancy Hypertens. 2013;3(2):86.

[60] Twig G, Hyde B and Shirihai OS. Mitochondrial fusion, fission and autophagy as a quality control axis: the bioenergetic view. Biochim Biophys Acta. 2008;1777(9):1092-7.

[61] Varanita T, Soriano ME, Romanello V, Zaglia T, Quintana-Cabrera R, Semenzato M, Menabo R, Costa V, Civiletto G, Pesce P, Viscomi C, Zeviani M, Di Lisa F, Mongillo M, Sandri M and Scorrano L. 
The OPA1-dependent mitochondrial cristae remodeling pathway controls atrophic, apoptotic, and ischemic tissue damage. Cell Metab. 2015;21(6):834-44.

[62] Kim CJ. Congenital lipoid adrenal hyperplasia. Annals of pediatric endocrinology \& metabolism. 2014;19(4):179-83.

[63] Olvera-Sanchez S, Espinosa-Garcia MT, Monreal J, Flores-Herrera O and Martinez F. Mitochondrial heat shock protein participates in placental steroidogenesis. Placenta.

2011;32(3):222-9.

[64] Foteinos $\mathrm{G}$ and $\mathrm{Xu} \mathrm{Q}$. Immune-mediated mechanisms of endothelial damage in atherosclerosis. Autoimmunity. 2009;42(7):627-33.

[65] Musial K, Szczepanska M, Szprynger K and Zwolinska D. The impact of dialysis modality on serum heat shock proteins in children and young adults with chronic kidney disease. Kidney \& blood pressure research. 2009;32(5):366-72.

[66] De los Rios Castillo D, Zarco-Zavala M, Olvera-Sanchez S, Pardo JP, Juarez O, Martinez F, Mendoza-Hernandez G, Garcia-Trejo JJ and Flores-Herrera O. Atypical cristae morphology of human syncytiotrophoblast mitochondria: role for complex $\mathrm{V}$. The Journal of biological chemistry. 2011;286(27):23911-9.

[67] Hanukoglu I, Rapoport R, Weiner L and Sklan D. Electron leakage from the mitochondrial NADPH-adrenodoxin reductase-adrenodoxin-P450scc (cholesterol side chain cleavage) system. Archives of biochemistry and biophysics. 1993;305(2):489-98.

[68] Black S, Kadyrov M, Kaufmann P, Ugele B, Emans N and Huppertz B. Syncytial fusion of human trophoblast depends on caspase 8. Cell death and differentiation. 2004;11(1):90-8.

[69] Huppertz B, Kadyrov M and Kingdom JC. Apoptosis and its role in the trophoblast. American journal of obstetrics and gynecology. 2006;195(1):29-39.

[70] Poidatz D, Dos Santos E, Gronier H, Vialard F, Maury B, De Mazancourt P and Dieudonne MN. Trophoblast syncytialisation necessitates mitochondrial function through estrogen-related receptorgamma activation. Molecular human reproduction. 2015;21(2):206-16.

[71] Shibata E, Nanri H, Ejima K, Araki M, Fukuda J, Yoshimura K, Toki N, Ikeda M and Kashimura M. Enhancement of mitochondrial oxidative stress and up-regulation of antioxidant protein peroxiredoxin III/SP-22 in the mitochondria of human pre-eclamptic placentae. Placenta. 2003;24(6):698-705.

[72] Ma K, Jin H, Hu R, Xiong $Y$, Zhou S, Ting $P$, Cheng $Y$, Yang $Y$, Yang $P$ and Li X. A proteomic analysis of placental trophoblastic cells in preeclampsia-eclampsia. Cell biochemistry and biophysics. 2014;69(2):247-58.

[73] Wang $Y$ and Walsh SW. Placental mitochondria as a source of oxidative stress in pre-eclampsia. Placenta. 1998;19(8):581-6.

[74] Muralimanoharan S, Maloyan A, Mele J, Guo C, Myatt LG and Myatt L. MIR-210 modulates mitochondrial respiration in placenta with preeclampsia. Placenta. 2012;33(10):816-23.

[75] Furui T, Kurauchi O, Tanaka M, Mizutani S, Ozawa T and Tomoda Y. Decrease in cytochrome c oxidase and cytochrome oxidase subunit I messenger RNA levels in preeclamptic pregnancies.

Obstetrics and gynecology. 1994;84(2):283-8.

[76] Hache S, Takser L, LeBellego F, Weiler H, Leduc L, Forest JC, Giguere Y, Masse A, Barbeau B and Lafond J. Alteration of calcium homeostasis in primary preeclamptic syncytiotrophoblasts: effect on calcium exchange in placenta. Journal of cellular and molecular medicine. 2011;15(3):654-67.

[77] Qiu C, Hevner K, Abetew D, Sedensky M, Morgan P, Enquobahrie DA and Williams MA. Mitochondrial DNA copy number and oxidative DNA damage in placental tissues from gestational diabetes and control pregnancies: a pilot study. Clin Lab. 2013;59(5-6):655-60.

[78] Muralimanoharan S, Maloyan A and Myatt L. Mitochondrial function and glucose metabolism in the placenta with gestational diabetes mellitus: role of miR-143. Clin Sci (Lond). 2016;130(11):93141. 
[79] Figueroa-Garcia Mdel C, Espinosa-Garcia MT, Martinez-Montes F, Palomar-Morales M and Mejia-Zepeda R. Even a Chronic Mild Hyperglycemia Affects Membrane Fluidity and Lipoperoxidation in Placental Mitochondria in Wistar Rats. PLoS One. 2015;10(12):e0143778. [80] Lindegaard ML, Damm P, Mathiesen ER and Nielsen LB. Placental triglyceride accumulation in maternal type 1 diabetes is associated with increased lipase gene expression. Journal of lipid research. 2006;47(11):2581-8.

[81] Shirasuna K, Takano H, Seno K, Ohtsu A, Karasawa T, Takahashi M, Ohkuchi A, Suzuki H, Matsubara S, Iwata $\mathrm{H}$ and Kuwayama T. Palmitic acid induces interleukin-1beta secretion via NLRP3 inflammasomes and inflammatory responses through ROS production in human placental cells. J Reprod Immunol. 2016;116:104-12.

[82] Mele J, Muralimanoharan S, Maloyan A and Myatt L. Impaired mitochondrial function in human placenta with increased maternal adiposity. Am J Physiol Endocrinol Metab. 2014;307(5):E419-25. [83] He L, Wang Z and Sun Y. Reduced amount of cytochrome c oxidase subunit I messenger RNA in placentas from pregnancies complicated by preeclampsia. Acta obstetricia et gynecologica Scandinavica. 2004;83(2):144-8.

[84] Qiu C, Hevner K, Abetew D, Sedensky M, Morgan P, Enquobahrie DA and Williams MA. Mitochondrial DNA copy number and oxidative DNA damage in placental tissues from gestational diabetes and control pregnancies: a pilot study. Clinical laboratory. 2013;59:655. 
Table 1. Changes in placental mitochondrial content in pregnancy pathologies.

\begin{tabular}{lll}
\hline Pathology & Results & Publication \\
\hline IUGR & Increased & Lattuada et al. (2008) [44] \\
IUGR + PE & Increased & \\
\hline IUGR & Decreased & Poidatz et al. (2015) [57] \\
IUGR + PE & Decreased & \\
\hline IUGR (whole tissue) & Increased & Mando et al. (2014) [51] \\
IUGR (cytotrophoblasts) & Decreased & \\
PE & No change & \\
\hline PE & Increased & Wang et al. (1998) [73] \\
\hline PE & Decreased & He et al. (2004) [83] \\
\hline PE (+IUGR) ${ }^{2}$ & Increased & Vishnyakova et al. (2016) [52] \\
\hline GDM & No Change & Qiu et al. (2013) [84] \\
\hline Type I DM & Nocreased & Mele et al. (2014) [82] \\
\hline Maternal obesity & No Change & \\
\hline Maternal obesity & Decreased & \\
\hline & & \\
\hline
\end{tabular}

\footnotetext{
${ }^{\mathrm{I}}$ Mando et al. (2014) found increased mitochondrial content in whole tissue but decreased mitochondrial content in isolated cytotrophoblasts

${ }^{2}$ IUGR reported in $61 \%$ of early onset PE and $27 \%$ of late onset PE

IUGR=intrauterine growth restriction; $\mathrm{PE}=$ preeclampsia; $\mathrm{DM}=$ diabetes mellitus; GDM=gestational diabetes mellitus
} 


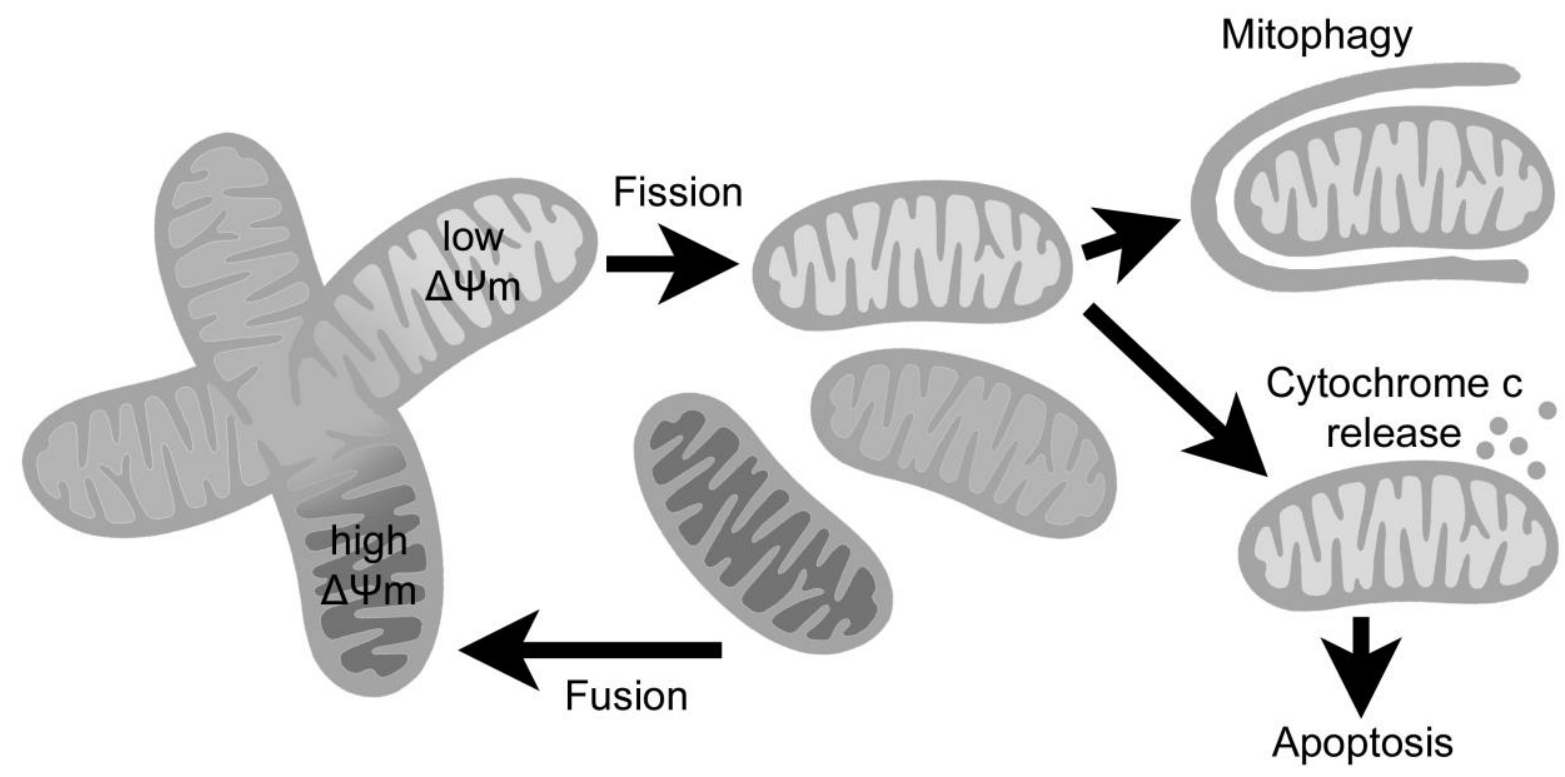

Figure 1. Model of mitochondrial regulation. Mitochondria form an interconnected network which is broken apart and reformed by the opposing forces of fission and fusion. Mitochondria with low membrane potential are targeted for mitophagy (a specialised form of autophagy targeting mitochondria), mitochondria with high membrane potential are more likely to fuse with other mitochondria. This balance allows the maintenance of a healthy pool of mitochondria with high membrane potential. If a large proportion of mitochondria are depolarised, this may lead to opening of the mitochondrial transition pore, release of inner membrane components such as cytochrome c, and apoptosis. $\Delta \Psi \mathrm{m}=$ mitochondrial membrane potential. 


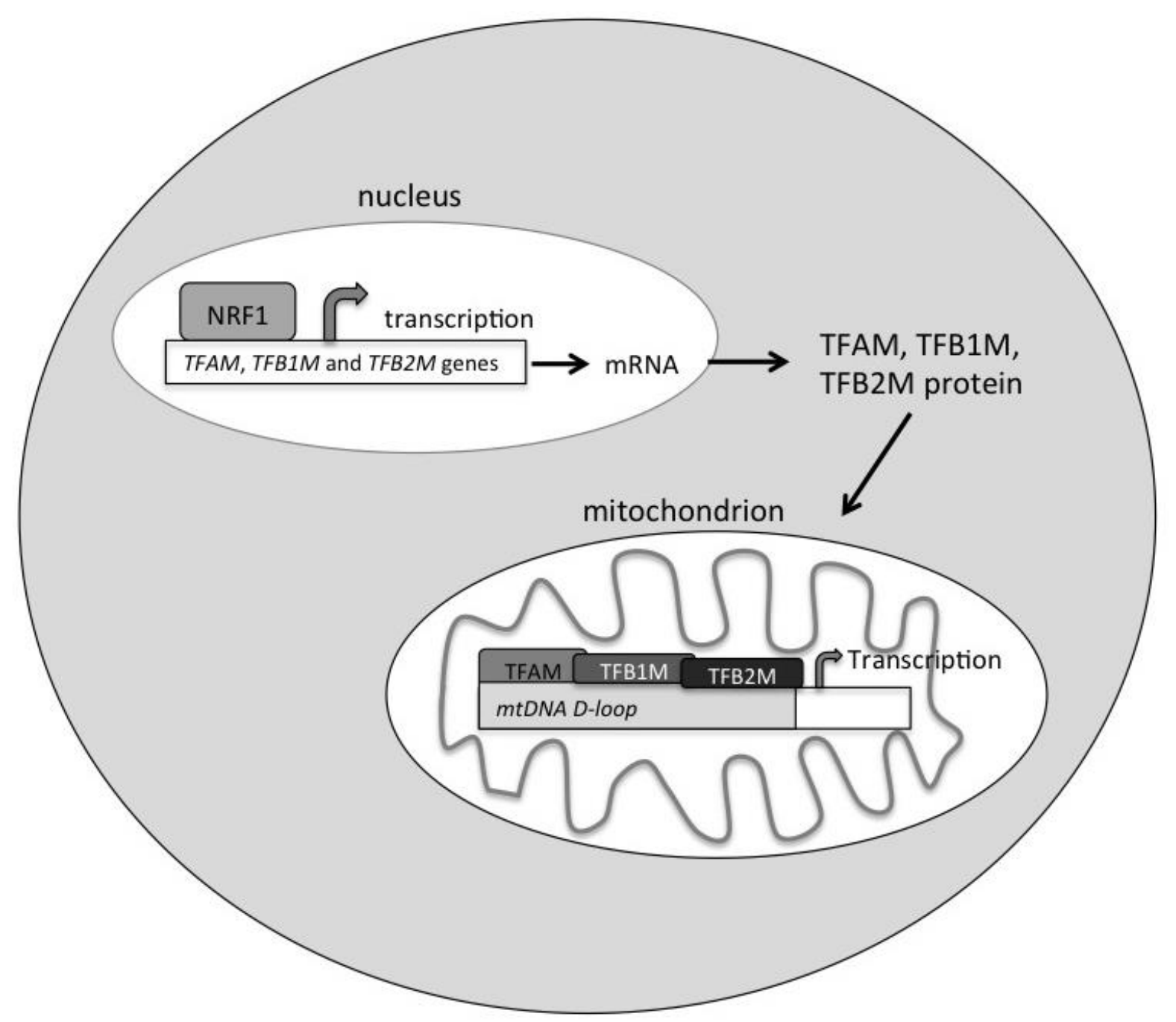

Figure 2. Control of mitochondrial biogenesis. Mitochondrial biogenesis is regulated by coordination of the nuclear and mitochondrial genomes. Upregulation of $N R F 1$ leads to production of TFAM, TFB1M and TFB2M which signal replication of the mitochondrial genome. NRF1=nuclear respiratory factor 1 ; TFAM=mitochondrial transcription factor A; TFB1M=mitochondrial transcription factor $\mathrm{B} 1$; TFB2M=mitochondrial transcription factor B2; mtDNA=mitochondrial DNA; D-loop=displacement loop. 


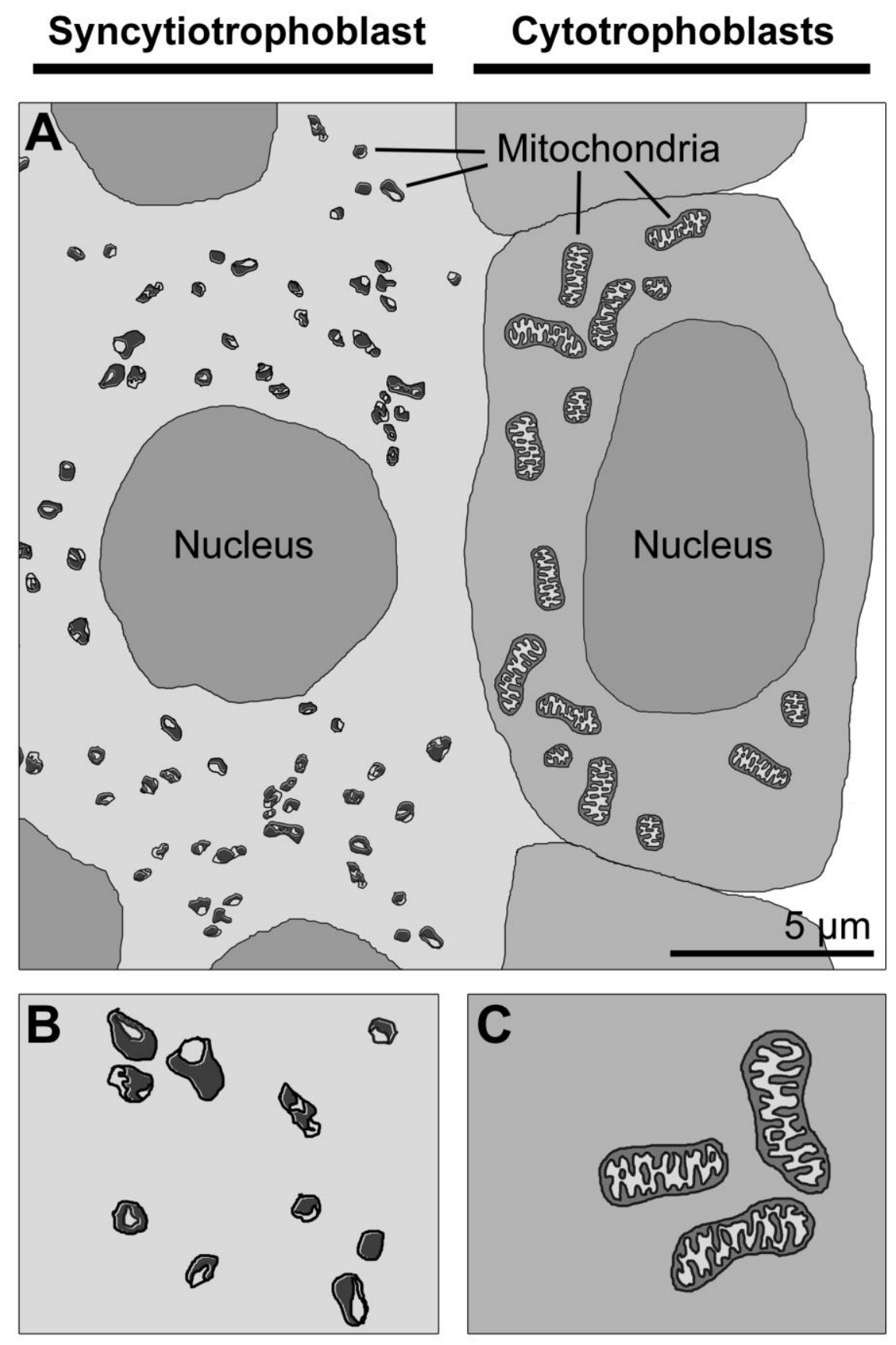

Figure 3. Syncytiotrophoblast and cytotrophoblast mitochondria. (A) Cartoon derived from published electron microscopy images $[53,66]$ depicting general structural features of mitochondria from the syncytiotrophoblast and cytotrophoblasts. (B) Mitochondria with the syncytiotrophoblast are small, and have a non-classical vesicular cristae structure and dense matrix. (C) Mitochondria with cytotrophoblasts are relatively larger than syncytiotrophoblast mitochondria and have a more typical cristae structure. 